\title{
Determinantes del desempeño académico de estudiantes universitarios de administración de empresas en Colombia
}

\author{
Eddy Fajardo $^{{ }^{\star}}$, Ligia Beleño-Montagut ${ }^{1}$, y Héctor Romero ${ }^{2}$ \\ (1) Departamento de Matemáticas y Ciencias Naturales, Universidad Autónoma de Bucaramanga, Avenida 42 No. 48 - \\ 11, Bucaramanga-Colombia (correo-e: efajardo@unab.edu.co*; Ibeleno@unab.edu.co) \\ (2) Escuela de Economía y Administración, Universidad Industrial de Santander, Cra. 27 calle 9, Bucaramanga- \\ Colombia (correo-e: hvalbuen@uis.edu.co)
}

* Autor a quién debe ser dirigida la correspondencia.

Recibido Jul. 15, 2021; Aceptado Sep. 20, 2021; Versión final Oct. 28, 2021, Publicado Feb. 2022

\begin{abstract}
Resumen
El presente estudio tiene como objetivo establecer los determinantes del desempeño académico de estudiantes universitarios del programa de administración de empresas (AE) en Colombia. La medición se hace a través de los resultados de las pruebas de estado (Saber Pro). Se recopila información de 143 instituciones de educación superior y de 28517 estudiantes en el año 2019. Se emplea el análisis de componentes principales como técnica de procesamiento de la información. Los resultados muestran que los estudiantes de la carrera de administración de empresas en un $65 \%$ son mujeres y en un $35 \%$ son hombres. Además, el $75 \%$ residen en la zona urbana y un $25 \%$ residen en la zona rural. Respecto a la modalidad de estudio, cerca de un $58 \%$ estudia de forma presencial, un $22 \%$ estudia a distancia y un $20 \%$ lo hace de forma virtual. Se concluye que existen diferencias regionales y brechas de género en las diferentes competencias que componen las pruebas académicas.
\end{abstract}

Palabras clave: administración; empresas; Saber Pro; factores socioeconómicos

\section{Determining factors of academic performance of business administration students in Colombia}

\begin{abstract}
The present study aims to identify determining factors that influence academic performance of business administration students in Colombia. Measurements are based on student results in the Colombian national exam (Saber Pro). Data is collected from 143 higher education institutions and from 28517 students in the year 2019. The principal component analysis method is used for data processing. The results show that $65 \%$ of business administration students are women and $35 \%$ are men. In addition, $75 \%$ of students reside in urban areas while $25 \%$ are from rural areas. Regarding study modes, the results reveal that $58 \%$ of students study in-person, $22 \%$ study via distance-education, and $20 \%$ study via virtual-education. It is concluded that there are geographical differences and gender gaps between students that affect their competency assessments in academic exams.
\end{abstract}

Keywords: business administration; Saber Pro; socioeconomic factors 


\section{INTRODUCCIÓN}

En la actualidad, la literatura académica ha centrado su atención en entender los efectos de la posición social, el género o la procedencia étnica en el logro académico de los estudiantes universitarios. Reconocer la incidencia de estos elementos resulta clave a la hora de garantizar una formación de calidad que permita que estos individuos sean capaces de agregar valor en las diferentes posiciones y acciones que emprendan, así como transformar en positivo a la sociedad en la que se desenvuelven. La disponibilidad y análisis de datos masivos de información, permite a los profesores ofrecer clases personalizadas y ajustadas a las necesidades de sus estudiantes, mientras que los estudiantes pueden identificar sus posibles problemas en el proceso de aprendizaje (Cao, 2021). Por lo general, para esa evaluación académica, es necesaria una prueba que permite obtener un resultado que mida el logro del estudiante conforme a unas competencias establecidas previamente.

Desde una mirada general, Takashiro y Clarke (2020), plantean que el desempeño académico de los estudiantes universitarios depende de factores que van desde el apoyo y los recursos materiales con las que cuentan sus familias, hasta su motivación y sus propias estrategias de aprendizaje. Algunos estudios destacan las condiciones socioeconómicas como principales determinantes del aprovechamiento de la educación superior por parte de los estudiantes, debido, en parte, a que con estos recursos económicos es que es posible acceder en mejores condiciones a la formación académica (Mountford-Zimdars et al., 2016; Wang y Liu, 2021; Cullinan et al., 2021). En el caso particular de China, Luo et al., (2018) encuentran que los estudiantes con un nivel de ingreso bajo no tienen acceso a una educación de calidad y llegan, incluso, a tener retornos económicos reducidos 0 , incluso, negativos.

La medición de la calidad en los estudios universitarios en Colombia se realiza a través de las pruebas de Estado Saber Pro, implementadas por el Instituto Colombiano para la Evaluación de la Educación (ICFES, 2020). En estas evaluaciones se identifica el conocimiento de los futuros profesionales en competencias genéricas y específicas. En el caso particular de los estudiantes de los programas de administración de empresas (AE) en Colombia, Rincón-Baez y Arias-Velandia (2019), destacan brechas de género en los resultados de las pruebas Saber Pro en los programas de AE, particularmente en competencias ciudadanas y en segundo idioma (inglés), a favor de los hombres. En un ámbito más amplio, relacionado con los programas de ciencias económicas, también persisten las diferencias de género en programas virtuales y a distancia; sólo que la brecha es menor (Arias-Velandia, et al., 2018).

Asimismo, los factores socioeconómicos como la ciudad de origen y los ingresos del núcleo familiar, entre otros, suelen guardar una relación directa. En el caso particular de Colombia, Viana et al., (2020) encuentra que el bajo aprovechamiento de los estudiantes en el área de administración de empresas está más asociado a las características propias de los participantes que a la institución de educación superior (IES). Además, los estudiantes de las IES privadas son más ineficientes, en términos de habilidades, que los de las IES del sector público. Estos elementos parecen explicar en buena medida la variabilidad en los resultados de las pruebas de Estado a nivel de educación superior, en el área de administración y programas relacionados. No obstante, Londoño et al. (2020), no encontraron evidencia suficiente de que los factores socioeconómicos tuviesen una incidencia significativa sobre los resultados en las pruebas de Estado en los estudiantes de AE. Dada esta evidencia empírica, es necesario profundizar los vínculos entre condiciones económicas y el desempeño académico.

La presente investigación se realiza sobre los resultados obtenidos en la prueba Saber Pro por parte de los estudiantes de $\mathrm{AE}$, una de las carreras con mayor demanda en el sistema educativo colombiano. Según cifras del Sistema Nacional de Información de la Educación Superior (SNIES, 2021), a corte de 2019, cerca del 7\% del total de estudiantes de Educación Superior en Colombia son del área de AE, por lo que se constituye como un programa del campo económico y administrativo con una demanda considerable. En este sentido surge la necesidad de establecer los determinantes del desempeño académico de los estudiantes de AE en Colombia en el año 2019. De esta forma, se podrán diseñar acciones y estrategias precisas que permitan mejorar la formación de un considerable número de estudiantes y así, contar con futuros profesionales idóneos, capaces de afrontar los retos empresariales del país y la región.

Para alcanzar este objetivo el estudio se divide de la siguiente forma: la presente sección, titulada como introducción, realiza una contextualización del fenómeno. Además, se describe la literatura académica reciente que permite identificar los aspectos más relevantes del problema de investigación. El siguiente apartado, la metodología de la investigación, detalla la muestra, variables y técnicas de procesamiento de información. Luego se presenta la sección de resultados, en donde se identifican los principales hallazgos, así como la discusión de estos. Por último, se ofrecen los comentarios finales. 


\section{METODOLOGÍA}

La población de este estudio pertenece a estudiantes de AE de las Instituciones de Educación Superior (IES) de Colombia que presentaron la Prueba Saber Pro en el año 2019. En total, se consideraron 28.517 estudiantes provenientes de 143 IES (ICFES, 2020). La distribución geográfica muestra que la mayoría de los estudiantes, tanto hombres como mujeres, pertenecen a los departamentos Antioquia, Cundinamarca, Valle del Cauca y Atlántico (ver figura 1). Por su parte, esta base de datos cuenta con variables cualitativas y cuantitativas clasificadas así: resultados de la prueba Saber Pro, características generales del estudiante, información sobre las IES y el desempeño académico. Las variables que se van a estudiar se describen en la tabla 1, donde se especifican las categorías a relacionar dentro del análisis: estudiante, familiar e IES.

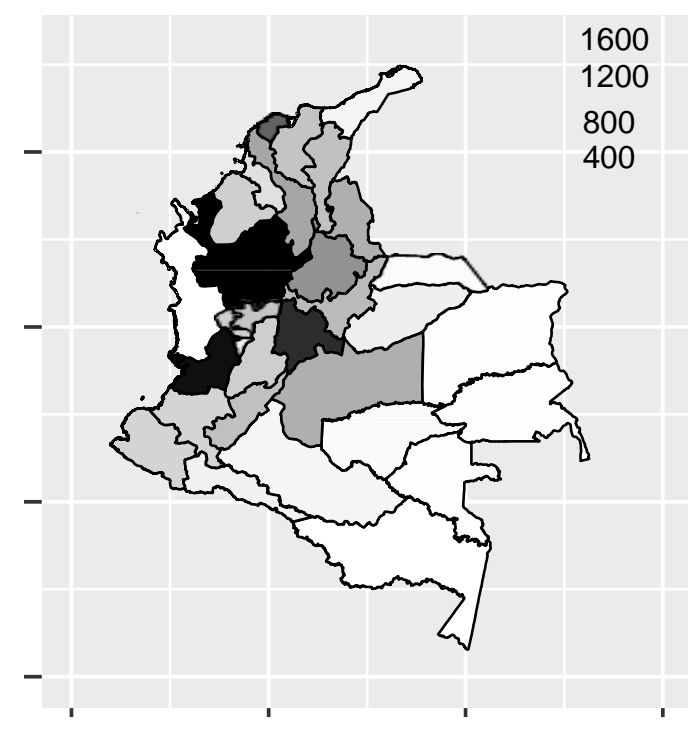

(a) Mujeres

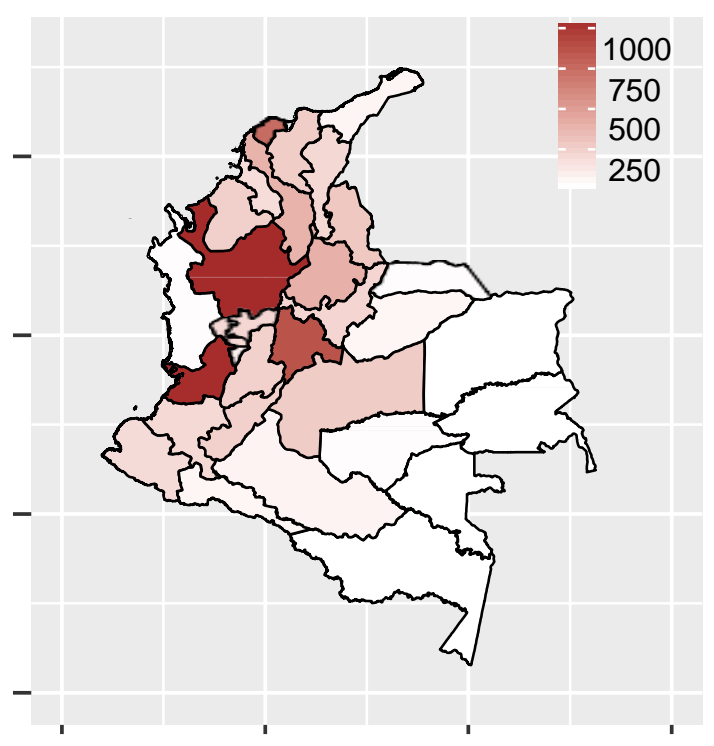

(b) Hombres

Fig. 1: Distribución espacial de los estudiantes en Colombia (2019) (Datos tomados del ICFES, 2020)

Tabla 1. Clasificación de las variables del estudio (Datos tomados del ICFES, 2020)

\begin{tabular}{|c|c|c|c|}
\hline $\begin{array}{l}\text { Característica } \\
\text { de la variable }\end{array}$ & Variable & Tipo & Valores \\
\hline \multirow[t]{4}{*}{ Estudiante } & Puntaje prueba & Cuantitativa & $0-300$ \\
\hline & Género & Cualitativa & Femenino - masculino \\
\hline & Edad & Cuantitativa & $20-70$ \\
\hline & Nivel socioeconómico (NSE) & Cualitativa & $1,2,3,4$ \\
\hline \multirow{2}{*}{ Familiar } & Área residencia & Cualitativo & Urbana - rural \\
\hline & Estrato & Cualitativo & Sin estrato, $1,2,3,4,5,6$ \\
\hline $\begin{array}{l}\text { Institución de } \\
\text { Educación } \\
\text { Superior (IES) }\end{array}$ & $\begin{array}{l}\text { Tipo } \\
\text { Modalidad } \\
\text { Carácter de la institución }\end{array}$ & $\begin{array}{l}\text { Cualitativa } \\
\text { Cualitativa } \\
\text { Cualitativa }\end{array}$ & $\begin{array}{l}\text { Pública - privada } \\
\text { Presencial, virtual y distancia } \\
\text { Institución tecnológica, Institución } \\
\text { universitaria, Tecnológica profesional, } \\
\text { Universidad }\end{array}$ \\
\hline
\end{tabular}

Para el tratamiento de la información se efectúa un análisis exploratorio para conocer el comportamiento de las variables. Además, mediante un análisis de componentes principales (ACP), se caracteriza y explica el rendimiento académico de los estudiantes universitarios teniendo en cuenta las variables contenidas en el conjunto original de datos con las demás variables suplementarias (cualitativas) consideradas en el estudio. El ACP es una técnica estadística que permite la reducción de dimensiones (número de variables) con la finalidad de explicar cómo es su comportamiento en el fenómeno de estudio. Al tener $n$ individuos con $p$ variables, la finalidad estará en reducir a un número más pequeño perdiendo poca información. El ACP es una combinación lineal de las variables cuantitativas originales que nos permite estudiar las interrelaciones entre éstas. De acuerdo con Peña (2002), el ACP es posible verlo como una recta que hace mínima las distancias ortogonales de los puntos a esta recta, por lo tanto, si se considera un punto $\mathrm{b} x_{i}$ y una trayectoria $\mathrm{k}_{1}=\left(\mathrm{k}_{11}, \ldots, \mathrm{k}_{1 \mathrm{p}}\right)^{\prime}$, determinada por un vector $\mathrm{k}_{1}$ cuyo módulo es la unidad, la proyección del punto $\mathrm{b}_{i}$ sobre esta trayectoria es el escalar:

$$
w_{i}=k_{11} b_{i 1}+\ldots+k_{1 p} b_{i p}=k_{1}^{\prime} b_{i}
$$


En la ecuación (1), el vector que muestra la proyección es $w_{i} k_{i}$. Siendo $r_{i}$ la distancia que hay entre el punto $b_{i}$, y su proyección sobre la dirección $k_{i}$, lo que involucra:

$$
\text { Minimizar } \sum_{i=1}^{n} \quad r_{i}^{2}=\sum_{i=1}^{n} \quad\left|b-w_{i} k_{i}\right|^{2}
$$

Al graficar $p$ puntos dimensionales con una pérdida mínima de información en un espacio de dimensión uno, es lo mismo que reemplazar las $p$ variables iniciales por una nueva variable, $w_{1}$, que abrevie perfectamente la información. Puede pensarse que la nueva variable tendrá en general una alta correlación con las variables originales. El software que se utiliza para el procesamiento y análisis de datos es R 4.0.4 (2021).

\section{RESULTADOS Y DISCUSIÓN}

El análisis descriptivo de los individuos de la prueba Saber Pro muestra que los estudiantes de la carrera de administración de empresas un $65 \%$ son mujeres y un $35 \%$ son hombres. Además, el $75 \%$ residen en la zona urbana y un $25 \%$ lo hace en la zona rural. Respecto a la modalidad de estudio cerca de un $58 \%$ estudia de forma presencial, un $22 \%$ estudia a distancia y un $20 \%$ lo hace de forma virtual. También se encontró que la mayoría de los universitarios pertenecen a los estratos 1, 2 y 3, y una menor proporción pertenecen a los estratos más altos (ver figura 2).

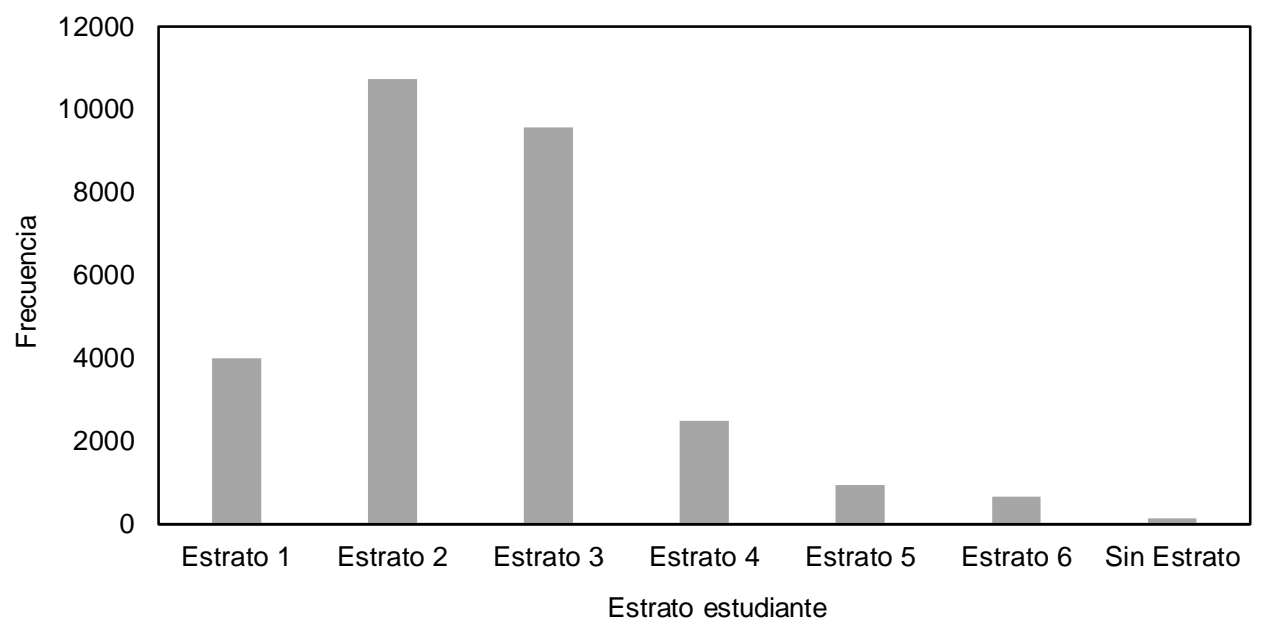

Fig. 2: Diagrama de barras según el estrato socioeconómico de los estudiantes (Datos tomados del ICFES, 2020)

En cuanto al estrato y el género del estudiante se encontró que las mujeres que estudian esta carrera son de recursos más limitados en comparación a los hombres. Este hallazgo está en sintonía con lo encontrado por Rincón-Baez y Arias-Velandia (2019) en relación con las diferencias de género existentes en los estudiantes de los programas de AE. En los estratos más altos se refleja una mayor igualdad económica entre los estudiantes sin importar el género (ver figura 3). A partir de la figura 3, también es posible aseverar que existe una mayor concentración de las mujeres en áreas administrativas y financieras, en parte por la amplia aceptación y penetración laboral en el país (Fajardo et al. 2018).

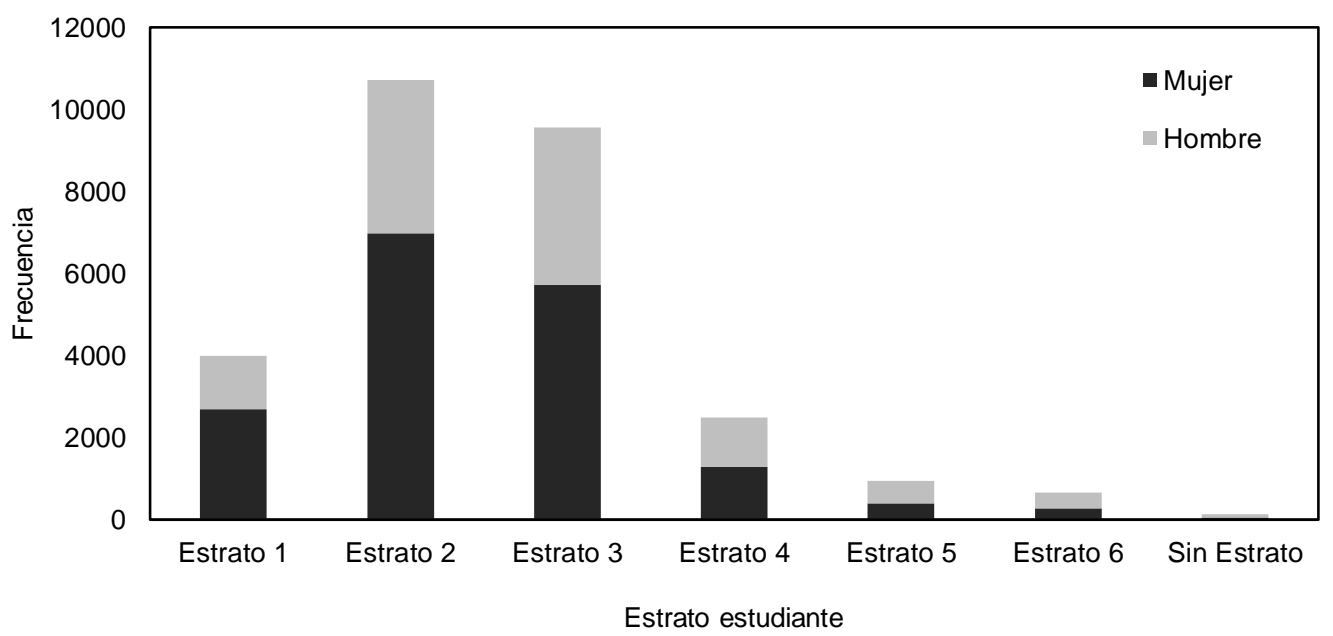

Fig. 3: Distribución de estudiantes según el estrato socioeconómico y su género (Datos tomados del ICFES, 2020) 
Teniendo en cuenta el carácter del tipo de IES, se halló que la mayoría de los universitarios pertenecen a instituciones universitarias o universidades, y una reducida proporción realizan sus estudios de esta carrera gerencial en instituciones técnicas o tecnológicas (ver figura 4).

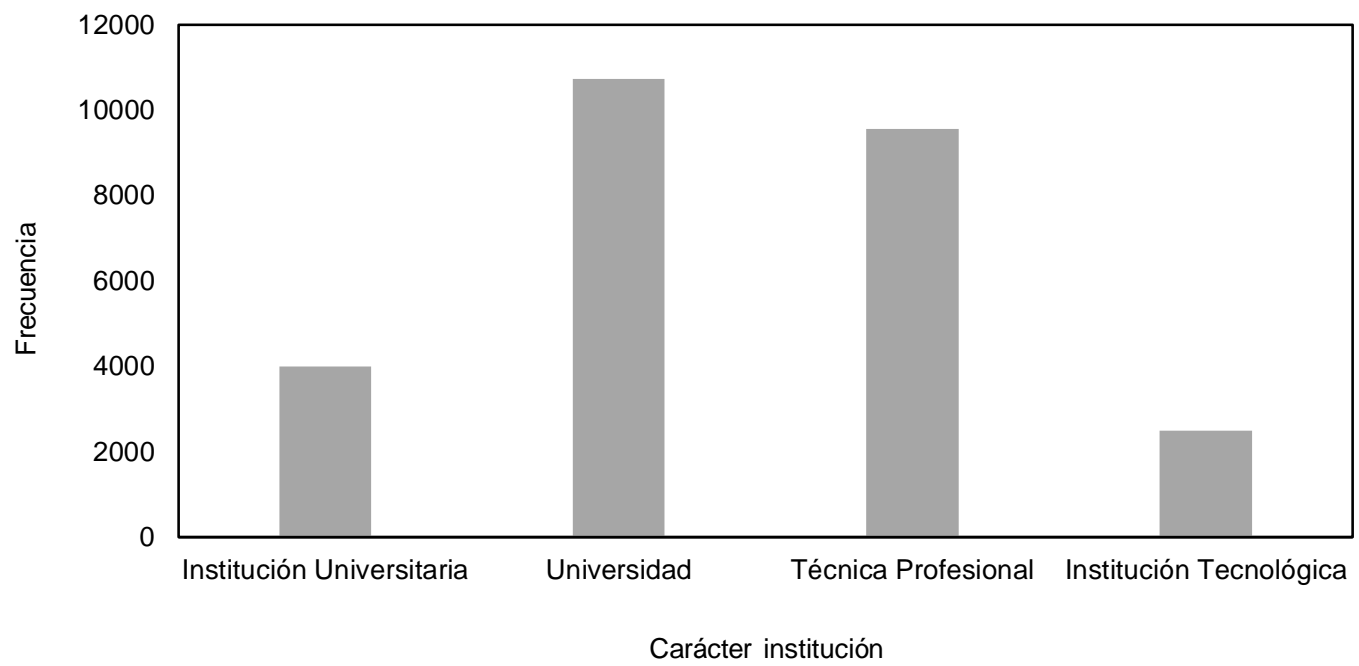

Fig. 4: Diagrama de barras del número de estudiantes según el carácter de la IES donde se encuentran matriculados (Datos tomados del ICFES, 2020)

En la figura 5, los diagramas de cajas y bigotes de las variables cuantitativas revelan que no hay diferencias significativas en la edad entre hombres y mujeres. Los resultados también muestran que respecto a los puntajes de la prueba Saber Pro existen diferencias en el módulo de razonamiento cuantitativo, inglés, competencias ciudadanas y lectura crítica según el género, siendo los hombres los que obtienen mejores resultados que las mujeres. Este hallazgo es particularmente relevante, pues destaca una ampliación de las brechas de género a la luz del estudio de Rincón-Baez y Arias-Velandia (2019). Esta diferencia se ve más marcada en los puntajes de razonamiento cuantitativo. En el caso del módulo comunicación escrita son similares los resultados entre hombres y mujeres.

Para determinar los factores del desempeño académico de los estudiantes de AE se hace uso del ACP. En la tabla 2 se muestran los valores propios y los porcentajes de inercia de los factores. Puede apreciarse que el primer factor capta un $51.57 \%$ y el segundo capta un $18.57 \%$. Estos dos factores captan aproximadamente el $70.14 \%$ de la variabilidad total del estudio. Por lo tanto, se realiza la selección de las dos primeras componentes.

Tabla 2. Valores propios e inercia explicada del desempeño académico de los estudiantes en las pruebas de Estado

\begin{tabular}{|c|c|c|}
\hline Valores propios & $\begin{array}{c}\text { Porcentaje } \\
\text { Inercia }\end{array}$ & $\begin{array}{c}\text { Porcentaje inercia } \\
\text { acumulada }\end{array}$ \\
\hline 2.58 & 51.57 & 51.57 \\
\hline 0.93 & 18.57 & 70.14 \\
\hline 0.59 & 11.82 & 81.96 \\
\hline 0.53 & 10.64 & 92.60 \\
\hline 0.37 & 7.40 & 100.00 \\
\hline
\end{tabular}

Teniendo en cuenta que si todas las variables contribuyeron por igual a la variabilidad captada por las componentes, cada una lo hace en un $20.0 \%$. Por lo tanto, la tabla 3 muestra que el primer componente está formado por el módulo de razonamiento cuantitativo (23.27\%), el módulo de lectura crítica (26.91\%), el módulo de competencia ciudadana (24.68\%) y el módulo de inglés (20.77\%). Por lo tanto, esta componente diferencia estudiantes con altos puntajes en estos módulos en la prueba Saber Pro de aquellos estudiantes con puntajes bajos. El segundo componente está compuesto por el módulo de comunicación escrita (95,41\%). Este componente distingue a los estudiantes con puntajes altos en este módulo de los estudiantes con puntajes bajos. 

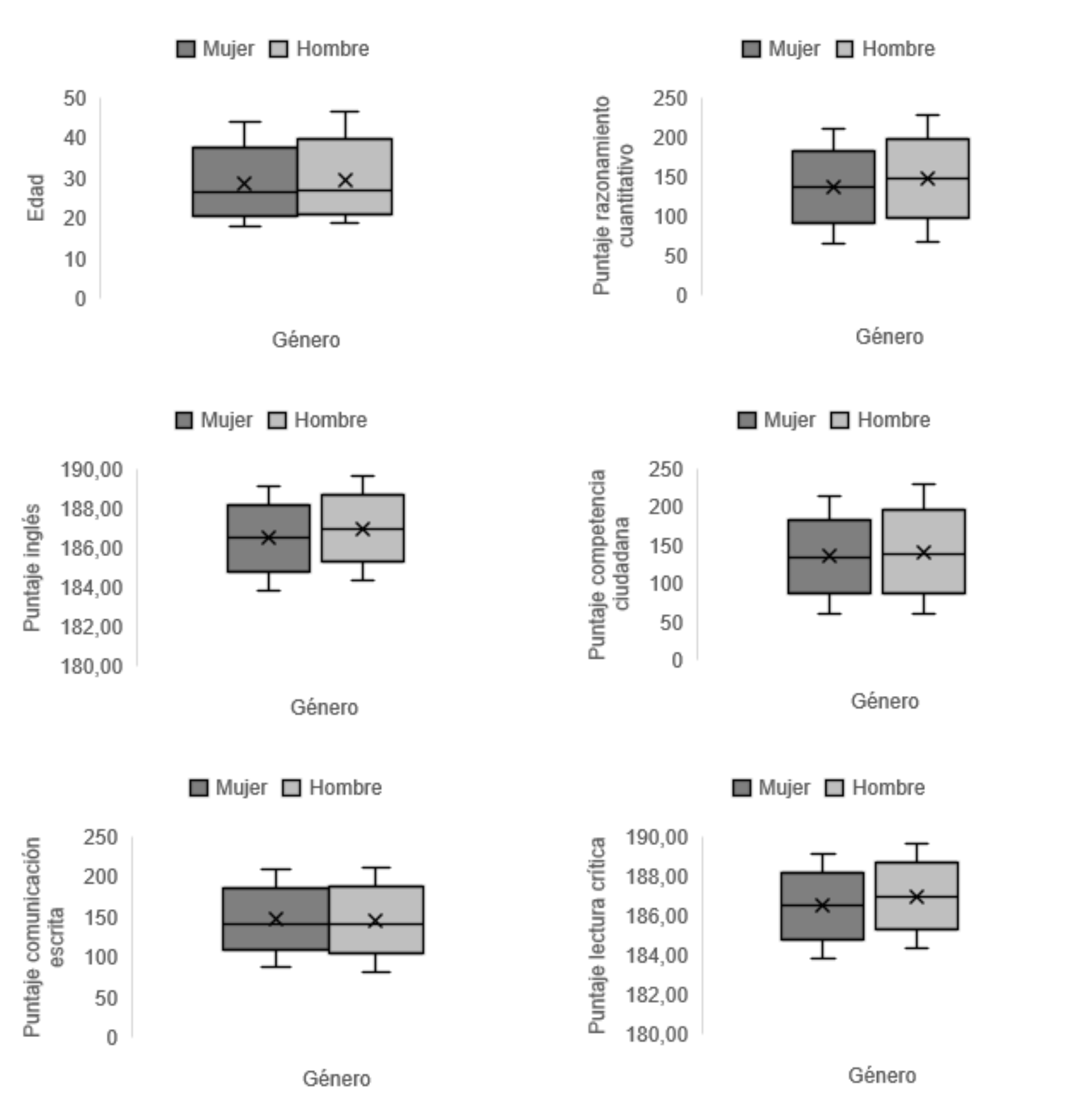

Fig. 5: Diagrama de cajas y bigotes de las variables: Género, edad y resultado en los diferentes módulos de la prueba Saber Pro (Datos tomados del ICFES, 2020)

Tabla 3. Contribución de las variables en el desempeño académico de los estudiantes

\begin{tabular}{|c|c|c|c|c|}
\hline Variable & $\begin{array}{c}\text { Componente } \\
1\end{array}$ & $\begin{array}{c}\text { Contribución componente } \\
1\end{array}$ & $\begin{array}{c}\text { Componente } \\
2\end{array}$ & $\begin{array}{c}\text { Contribución componente } \\
1\end{array}$ \\
\hline $\begin{array}{c}\text { Razonamiento } \\
\text { Cuantitativo }\end{array}$ & 0.78 & $23.27 \%$ & -0.11 & $1.24 \%$ \\
\hline Lectura crítica & 0.83 & $26.91 \%$ & -0.13 & $1.82 \%$ \\
\hline Competencia ciudadana & 0.80 & $24.68 \%$ & -0.11 & $1.19 \%$ \\
\hline Inglés & 0.73 & $20.77 \%$ & -0.06 & $0.34 \%$ \\
\hline Comunicación escrita & 0.34 & $4.38 \%$ & 0.94 & $95.41 \%$ \\
\hline
\end{tabular}


Las variables suplementarias permiten ver en el componente 1 que estudiantes de género maculino, con un nivel económico alto, que viven en el área urbana, de estratos altos, con modalidad de la carrera presencial, matriculados en universidades, obtienen mejores resultados en los módulos de razonamiento cuantitativo, lectura crítica, competencia ciudadana e inglés. Esto está en sintonía con lo encontrado por Cayón, SantiagoCorrea y Scarpetta (2021) quienes afirman que los estudiantes provenientes de la Unión de Colegios Internacionales (UNICOL) tienen mejor desempeño en las pruebas estandarizadas. Por lo general, estas instituciones educativas están conformadas por hogares de altos ingresos. Por el contrario, las mujeres, con nivel económico bajo, que residen en la zona rural, de estratos bajos, con modalidad virtual o a distancia y matriculadas en instituciones tecnológicas o universitarias, obtienen resultados bajos en estos mismos módulos (ver tabla 4).

Tabla 4. Significancia de las variables suplementarias del desempeño académico (codificación de la significancia estadística: ${ }^{* *}$ a $0.001,{ }^{* *}$ a $0.01,{ }^{*}$ a 0.05, ' ' a 0.1 )

\begin{tabular}{|c|c|c|c|c|c|}
\hline Variable & Categoría & Componente 1 & $\begin{array}{c}\text { Estadístico } \\
\text { Componente } 1\end{array}$ & Componente 2 & $\begin{array}{c}\text { Estadístico } \\
\text { Componente } 2\end{array}$ \\
\hline \multirow[t]{2}{*}{ Género } & Femenino & $-0.13^{* * \star}$ & -17.77 & $0.06^{\star \star \star}$ & 12.23 \\
\hline & Masculino & $0.21^{\star \star \star}$ & 17.77 & $-0.09^{\star \star \star}$ & -12.23 \\
\hline \multirow[t]{4}{*}{ NSE } & 1 & $-0.45^{\star \star \star}$ & -20.47 & $0.05^{\star \star}$ & 3.43 \\
\hline & 2 & $-0.24^{* \star *}$ & -18.95 & $0.01^{*}$ & 1.57 \\
\hline & 3 & $-0.10^{\star \star}$ & -3.837 & -0.01 & -0.58 \\
\hline & 4 & $0.46^{\star \star \star}$ & 36.94 & $-0.03^{* *}$ & -3.75 \\
\hline \multirow[t]{2}{*}{ Área residencia } & Rural & $-0.44^{\star \star \star}$ & -18.81 & $0.02^{\star}$ & 1.68 \\
\hline & Urbana & $0.07^{\star \star *}$ & 18.81 & $-0.00^{*}$ & -1.68 \\
\hline \multirow[t]{7}{*}{ Estrato } & Sin Estrato & $-0.61^{* *}$ & -4.42 & -0.12 & -1.40 \\
\hline & 1 & $-0.55^{\star \star \star}$ & -23.14 & 0.02 . & 1.62 \\
\hline & 2 & $-0.19^{\star \star \star}$ & -15.34 & $0.02^{\star}$ & 2.56 \\
\hline & 3 & $0.07^{\star \star}$ & 5.31 & $-0.03^{*}$ & -3.33 \\
\hline & 4 & $0.61^{* \star *}$ & 19.83 & -0.00 & -0.25 \\
\hline & 5 & $1.09^{\star \star \star}$ & 21.30 & -0.02 & -0.53 \\
\hline & 6 & $1.58^{\star \star *}$ & 25.57 & 0.01 & 0.21 \\
\hline \multirow[t]{3}{*}{ Modalidad } & Distancia & $-0.49^{* \star *}$ & -26.76 & 0.01 & 0.96 \\
\hline & Virtual & $-0.13^{* *}$ & -6.40 & $0.03^{*}$ & 2.41 \\
\hline & Presencial & $0.21^{* \star *}$ & 27.50 & $-0.01^{*}$ & -2.71 \\
\hline \multirow[t]{4}{*}{$\begin{array}{l}\text { Carácter } \\
\text { institución }\end{array}$} & Institución Tecnológica & $-0.55^{\star \star}$ & -7.83 & -0.05 & -1.19 \\
\hline & Institución Universitaria & $-0.21^{* \star *}$ & -23.12 & 0.01 . & 1.85 \\
\hline & $\begin{array}{l}\text { Tecnológica } \\
\text { Profesional }\end{array}$ & $-0.82^{* \star \star}$ & -22.51 & $0.08^{\star \star}$ & 3.44 \\
\hline & Universidad & $0.43^{\star \star *}$ & 36.92 & $-0.02^{* \star}$ & -3.28 \\
\hline
\end{tabular}

Ahora, las variables suplementarias en el componente 2 muestran que las mujeres con un nivel económico muy bajo, de estrato 2, que estudian en modalidad virtual en instituciones universitarias o tecnológicas profesionales, obtienen mejores resultados en el módulo de comunicación escrita en comparación con los hombres (ver tabla 4). Por lo tanto, el ACP permite afirmar que los estudiantes de la carrera de administración de empresas en Colombia que obtienen resultados más bajos en todos los módulos de la prueba Saber Pro son aquellos que viven en estratos bajos y estudian en instituciones universitarias o instituciones tecnológicas.

\section{CONCLUSIONES}

El estudio realizado muestra que la mayoría de los estudiantes de la carrera de AE son de género femenino, residen en el área urbana, asisten de forma presencial a la universidad, son de estratos 1,2 y 3 y están matriculados en universidades e instituciones universitarias. Además, se encontró que los hombres obtienen mejores resultados en los módulos de razonamiento cuantitativo, inglés, competencias ciudadanas y lectura crítica en comparación a las mujeres. Este es un hallazgo que deja entrever la posibilidad de que exista un sesgo en la formación basado en el género, que solía estar representado; según estudios previos, en el ámbito 
de las ingenierías, concretamente en la ingeniería civil (Cantillo y García, 2014). En este sentido, contar con espacios y entornos que permitan reducir estas brechas en el aprendizaje, entre hombres y mujeres, puede resultar fundamental (Arias-Velandia, Rincón-Báez, y Cruz-Pulido, 2018). El análisis de componentes principales mostró que el desempeño académico de los universitarios de género masculino, con un nivel económico alto, que viven en el área urbana, de estratos altos, con modalidad de la carrera presencial, matriculados en universidades, es bueno en la prueba Saber Pro comparado con las mujeres.

\section{AGRADECIMIENTOS}

Los autores agradecen el apoyo financiero otorgado por la Universidad Autónoma de Bucaramanga (UNAB) a través del proyecto de investigación titulado "Determinantes del desempeño académico de los estudiantes de administración de empresas en las pruebas Saber Pro en el 2019" bajo el acta 084.

\section{REFERENCIAS}

Arias-Velandia, N., Rincón-Báez, W., y Cruz-Pulido, J.M., Women and men's performance in face-to-face, virtual and distance higher education in Colombia, http://dx.doi.org/10.15765/pnrm.v12i22.1142, Revista Panorama, 12(22), 58-69 (2018)

Cantillo, V., y García, J., Gender and other factors influencing the outcome of a test to assess quality of education in Civil Engineering in Colombia, 10.1061/(ASCE)El.1943-5541.0000194, Journal of Professional Issues in Engineering Education and Practice, 140(2), 2-33 (2014)

Cao, Y., Portrait-based academic performance evaluation of college students from the perspective of Big Data, https://doi.org/10.3991/ijet.v16i04.20475, International Journal of Emerging Technologies in Learning, 16(04), 95-106 (2021)

Cayón, E., Santiago-Correa, J., y Scarpetta, L., Caracterización de los programas académicos y determinación de las variables que afectan el rendimiento de los estudiantes en el Colegio de Estudios Superiores de Administración, CESA, http://dx.doi.org/10.4067/S0718-50062021000500175, Formación Universitaria, 14(5), 175-186 (2021)

Cullinan, J., Flannery, D., y otros tres autores, The disconnected: COVID- 19 and disparities in access to quality broadband for higher education students, https://doi.org/10.1186/s41239-021-00262-1, International Journal of Educational Technology in Higher Education, 18(26) (2021)

Fajardo, E., Romero, H., Álvarez, M., y Vargas, J.M., Participation of women in the labor market in Colombia: challenges and recent trends, Revista Espacios, ISSN: 0798 1015, 39(48), 12 (2018)

ICFES, Instituto Colombiano para la Evaluación de la Educación, Investigación, https://www.icfes.gov.co/ (2020)

Luo, Y., Guo, F., y Shi, J., Expansion and inequality of higher education in China: how likely would Chinese poor students get to success?, 10.1080/07294360.2018.1474856, Higher Education Research and Development, 37(5), 10151034 (2018)

Londoño, E., Ahumada, V., Gutiérrez, D., y Gamboa, M., Conductores socioeconómicos sobre la lectura crítica según las pruebas Saber Pro en Colombia 2015, http://dx.doi.org/10.4067/S0718-50062020000400045, Formación Universitaria, 13(4), 45-56 (2020)

Mountford-Zimdars, A., Moore, J., y Graham, J., Is contextualised admission the answer to the access challenge?, https://doi.org/10.1080/13603108.2016.1203369, Perspectives: Policy and Practice in Higher Education, 20(4), 143-150 (2016)

Peña, D., Análisis de datos multivariantes, Editorial McGraw-Hill Interamericana, ISBN: 978-8-44-813610-9 Madrid, España (2002)

Rincón-Baez, W., y Arias-Velandia, N., Brecha de rendimiento académico por género en Saber Pro en programas de administración en los departamentos de Colombia, https://doi.org/10.15765/pnrm.v13i25.1410, Revista Panorama, 13(25), 142-161 (2019)

SNIES, Sistema Nacional de Información de Educación Superior, Estadísticas, https://snies.mineducacion.gov.co/portal/ (2021)

Takashiro, N., y Clarke, C., Low-socioeconomic status students turn their academic failure to success: a synthesis of qualitative research, in Mistakes, errors, and failures across cultures by E. Vanderheiden y C. Mayer (Editors), 363-382, Springer Nature, ISBN: 978-3-030-35574-6, Switzerland (2020)

Viana, R.A., Arranz, J.M., y García-Serrano, C., Efficiency of university education: a partial frontier analysis, https://doi.org/10.47872/laer-2020-29-1s, Latin American Economic Review, 29, 2-16 (2020)

Wang, L., y Liu, C., Lost in mobile? Exploring the mobile internet digital divide among Chinese college students, https://doi.org/10.1186/s41239-021-00267-w, International Journal of Educational Technology in Higher Education, 18(31) (2021) 